\title{
Linx
}

Revue des linguistes de l'université Paris X Nanterre

$8 \mid 1996$

Du dire et du discours

\section{De l'enquête à l'édition : parcours douloureux mais essentiel pour une analyse de discours oraux}

\section{Sandrine Wachs}

\section{(2) OpenEdition}

\section{Journals}

Édition électronique

URL : http://journals.openedition.org/linx/1176

DOI : 10.4000/linx.1176

ISSN : 2118-9692

\section{Éditeur}

Presses universitaires de Paris Nanterre

\section{Édition imprimée}

Date de publication : 1 septembre 1996

Pagination : 187-206

ISSN : 0246-8743

\section{Référence électronique}

Sandrine Wachs, « De l'enquête à l'édition : parcours douloureux mais essentiel pour une analyse de discours oraux », Linx [En ligne], 8| 1996, mis en ligne le 17 juillet 2012, consulté le 06 mai 2019. URL http://journals.openedition.org/linx/1176 ; DOI : 10.4000/linx.1176

Ce document a été généré automatiquement le 6 mai 2019.

Département de Sciences du langage, Université Paris Ouest 


\title{
De l'enquête à l'édition : parcours douloureux mais essentiel pour une analyse de discours oraux
}

\author{
Sandrine Wachs
}

1 De la même façon que l'analyse de discours nous « conduit à découvrir l'hétérogénéité constitutive d'un discours généralement considéré comme lisse et monolithique $»^{1}$, la transcription de discours oraux nous amène à découvrir l'hétérogénéité organisée d'un oral généralement considéré comme désordonné et incohérent.

2 Etudier l'oral de façon systématique suppose qu'on en fasse un objet d'analyse. Il faut donc commencer par le rendre accessible - le noter - contrairement à l'écrit qui peut se travailler tel quel : «Ce non-sens indispensable, donner à 'voir', par la transcription, ce que la machine a enregistré comme ayant été dit, procure à chacun, quel qu'il soit, une blessure mal analysée $»^{2}$. L'observation doit obligatoirement passer par l'écriture, ne serait-ce que parce que notre mémoire auditive est très faible. De plus, tant qu'on n'a pas transposé à l'écrit, on ne peut avoir conscience des spécificités de l'oral, et en particulier de la régularité avec laquelle certains phénomènes apparaissent. Incontournable paradoxe: une écriture peu réfléchie des données orales peut parfois conduire au "camouflage" de ses propres spécificités ${ }^{3}$.

3 «Pas d'avancée en linguistique sans ce questionnement sur l'oral et pas d'oral sans enquête, corpus, témoins $»^{4}$. Noter l'oral suppose en effet tout un travail de recueil puis d'écoute, deux passages incontournables que nous nous proposons de décrire avant d'aborder l'étape ultime du travail pour une analyse de discours oraux : la notation. On verra que le travail d'analyse ne commence pas à l'écriture mais qu'il s'élabore lentement dès le recueil des données. En ce sens, la transcription n'est pas réductible à un élément empirique de l'analyse : elle devient un élément constitutif de l'analyse proprement dite. 


\section{Le recueil des données orales}

4 Recueillir de l'oral nous confronte immédiatement à divers problèmes posés par l'enquête. D'une façon très générale, deux remarques s'imposent à ce sujet :

5 - On ne recueille pas les données au hasard, mais chacun adapte - bien souvent intuitivement - sa méthode d'enquête au type de description souhaitée. Selon qu'on travaille en phonétique, en phonologie, en syntaxe, en analyse de discours, etc., l'enquête ne se mènera pas de la même manière ${ }^{5}$. En analyse de discours, par exemple, le recueil des données est envisagé d'une façon beaucoup plus large qu'en phonologie ou même qu'en syntaxe : on cherche à récupérer les incidences du situationnel et du contextuel dans la production orale en tenant compte de la multidimensionnalité du langage oral (position des locuteurs, gestes, attitudes.... ${ }^{6}$.

6 Avant même que se posent les choix liés aux conventions de transcription, c'est dès l'étape de recueil des données qu'il faut opter pour une méthodologie en fonction du but de la recherche.

7 - Quand on part sur le terrain, on se retrouve très souvent confronté au célèbre "paradoxe de l'observateur" défini par Labov7?. Il faut en effet pouvoir garder une distance par rapport à l'objet étudié tout en restant très proche de celui-ci.

8 On se propose de passer rapidement en revue tous les phénomènes qui touchent à l'enquête au sens large, c'est-à-dire tout paramètre susceptible de conditionner les données orales. Le but recherché dans cette étape de recueil serait d'aboutir à une sorte de fiche générale sur laquelle figureraient des informations relatives à l'enquête telles que le lieu, les locuteurs, le type d'oral recueilli ${ }^{8}$; l'intention étant de rendre les corpus facilement accessibles aux différents chercheurs 9 .

9 On sait que le recueil des données varie en fonction des objectifs. En précisant dès le départ l'optique de travail, on explique déjà certains des choix futurs selon qu'il s'agit d'une étude phonétique, syntaxique, sociolinguistique, discursive, plutôt orientée vers une analyse strictement linguistique ou plutôt pédagogique...

10 D'une façon générale, il est préférable que celui qui analyse soit celui qui non seulement transcrive mais également recueille: être sur le terrain permet de photographier la situation, donc d'emmagasiner un grand nombre d'informations qui peuvent s'avérer très utiles lors du passage à l'écriture ${ }^{10}$.

11 Lieu où on se déplace pour recueillir les données, le terrain mérite une description détaillée : la production orale se nourrit sans cesse du contexte situationnel, de ce que François regroupe sous l'appellation «ensemble sémiologique d'un acte de communication» ${ }^{11}$. Préciser le lieu : extérieur (jardin, square public, rue, etc.) ou intérieur (maison, école, magasin, hôpital, etc.) permet, en situant les conditions de l'échange (plutôt calme ou plutôt bruyant), d'expliquer certains phénomènes tels que des passages inaudibles par exemple. De plus, informer du type de relation instaurée entre l'enquêteur et l'enquêté (ou entre les informateurs eux-mêmes s'il y a lieu) permet de restituer le climat de l'entretien : plutôt tendu/détendu.

12 Le terrain idéal serait - selon Labov ${ }^{12}$ - le milieu naturel de l'informateur : l'enquêteur a tout à gagner à se déplacer dans le lieu de vie de l'informateur. Perdu dans un milieu étranger au sien, tout locuteur aura tendance à se protéger soit en se surveillant fortement, soit en se bloquant. Il est alors facile d'imaginer les conséquences résultant 
d'une telle situation ${ }^{13}$. Dans le cas où le chercheur n'est pas en mesure de recueillir luimême les données qu'il compte travailler, Labov a proposé la méthode de «l'observation participante » qui utilise des observateurs issus du milieu étudié ${ }^{14}$. Cette méthode permet de contourner le problème de la crédibilité de l'enquêteur dans certains milieux sociaux en même temps que ce que Labov avait appelé « le paradoxe de l'observateur ».

Les informateurs doivent faire l'objet d'une description détaillée : en précisant le nombre, on saura par exemple qu'on peut trouver des chevauchements de paroles ${ }^{15}$; en indiquant le degré de familiarité entre enquêteur/enquêté(s) - inconnus, simples connaissances, intimes - on sera informé sur le degré de connivence existant entre les deux (ou plusieurs) membres de l'échange : plus la confiance est installée entre les protagonistes, moins la conversation est surveillée. Dans un registre plus spécifiquement sociolinguistique, il est tout aussi intéressant de préciser le statut social (scolarité, lieu de résidence, profession et celle des parents - déterminante pour définir la mobilité sociale vers le haut ou vers le bas au sein de la famille), l'âge, le sexe, ainsi que l'origine géographique des locuteurs. Toutes ces informations peuvent être déterminantes pour la suite de l'analyse et/ou pour le chercheur utilisateur de corpus ne lui appartenant pas.

On peut très rapidement évoquer le choix du matériel qui se fait en fonction du but de la recherche. Selon qu'on travaille en phonétique, en syntaxe, en analyse du discours... le matériel - ainsi que les conditions d'enregistrement - ne seront pas forcément les mêmes : "Les exigences techniques dépendent en partie des objectifs qu'on a fixés à la recherche", tout en sachant que "[...] une technique très riche va de pair avec un appauvrissement de la situation de paroles $»^{16}$.

Enfin, indiquer la provenance des données sur lesquelles on travaille (enregistrement de radio, de télévision, ou données recueillies sur le terrain), le type d'oral recueilli (conversation familière, entretien directif ou semi-directif, lecture de texte ou de liste de mots...) ainsi que le contenu de l'échange (narration, explication, introspection ...) permet de cibler dès le départ le type d'oral auquel on aura à faire.

On aura compris que l'étape du recueil des données est tout à fait déterminante pour la suite du parcours jusqu'à l'analyse: elle ne se fait pas au hasard mais en fonction des objectifs de recherche propres à chacun. S'appliquer à aménager une "carte d'identité" détaillée de chaque corpus permettrait une utilisation plus large de ces corpus, jusque-là très peu exploités ${ }^{17}$.

\section{L'écoute}

Passer à l'écriture de ces données suppose qu'on apprenne à les écouter. Le problème est que toute transcription est une approximation de ce qui a réellement été produit, en partie parce que l'oreille est trompeuse et qu'on tend à percevoir ce qui correspond à nos attentes, en fonction de la norme ${ }^{18}$. Notre oreille, entraînée à reconnaître des morphèmes, aura beaucoup de difficultés à entendre des "sons" : «On n'écoute pas des sons ni des éléments isolés, mais, comme l'ont observé tous les spécialistes de la perception, des séquences auxquelles on donne un sens $»^{19}$. Concrètement, on n'écoute pas un enregistrement en laissant de côté les préjugés qu'on a sur la langue, surtout lorsqu'il s'agit de notre langue maternelle. Il en va de même pour les préjugés qu'on s'est construits sur les informateurs au moment de l'échange. On peut par exemple s'attendre à ce qu'un locuteur, perçu comme cultivé, "parle mieux" qu'un locuteur de "français 
populaire"20. Une des plus grandes réussites de la sociolinguistique est d'avoir su démontrer que la réalité est beaucoup plus complexe, particulièrement en dégageant l'hétérogénéité de toute communauté linguistique, et de tout idiolecte ${ }^{21}$.

D'un point de vue plus "technique", il y a toujours décalage entre écoute directe et écoute enregistrée : l'enregistrement n'est qu'une imitation atrophiée, sans relief, de la réalité sonore. Les sons y sont comme écrasés, surtout lorsque les conditions d'enregistrement n'ont pas été optimisées. Dans les faits, l'écoute est rendue difficile par la rapidité avec laquelle les énoncés s'enchaînent et se superposent.

Le travail d'écoute est continuellement parsemé de pièges auditifs : on est toujours en proie à ce que Stubbs appelle des «hallucinations auditives» ${ }^{22}$. Pour faire face aux problèmes d'écoute, Blanche-Benveniste et Jeanjean (1987) ont préconisé le travail à plusieurs transcripteurs. Si cela permet d'être plus précis et de mieux négocier les pièges tendus par l'écoute, cela aboutit souvent à plusieurs interprétations pour un même passage.

20 La configuration des multi-interprétations la plus courante est celle où les deux transcripteurs entendent le même nombre de syllabes. Cela se produit très fréquemment à propos du timbre d'une voyelle $([a] / \mathrm{a}] ;[\tilde{\varepsilon}] /[a] ;[\tilde{\varepsilon}] /[e] ;$ etc.), ou de la nature d'une consonne ([aveglyi] / [aveklui]; [pa] / [twa]) par exemple ${ }^{23}$, ce qui peut parfois aboutir à de graves écarts interprétatifs sur le plan grammatical. L'exemple suivant fait apparaître une relative dans la première interprétation uniquement :

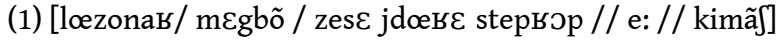

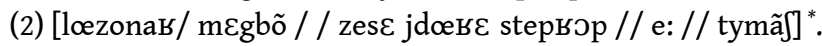

* (1) le zonard/mec(s) bon / zesayent de rester propres // et // qui marche(nt)

(2) le zonard/mec(s) bon / zesayent de rester propres // et // tu marches.

21 Les écarts d'interprétation peuvent parfois être très impressionnants. Il faut se faire une raison. Il est effectivement difficile d'accepter que là où on entend d'abord quelqu'un d'autre entende là-bas ${ }^{24}$. On a même rencontré des situations où la différence d'écoute entre les deux transcripteurs apparaissait comme "monstrueuse" :

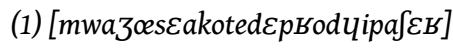

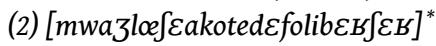

${ }^{*}(1)$ moi je sais [akote] des produits par chers

(2) moi [3loef à côté des Folies Bergères.

où il est difficile de savoir si le locuteur sait rapporter des produits pas chers ou s'il logeait ${ }^{25} \grave{a}$ côté des Folies Bergères... Bien entendu, il nous était impossible de revenir sur nos jugements respectifs, même en laissant passer plusieurs mois entre les différentes écoutes.

On peut aller encore plus loin dans la série des "hallucinations auditives": il arrive parfois que deux écoutes ne fassent pas ressortir le même nombre de syllabes. Par exemple :

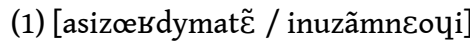

(2) [asizœbdymat $\tilde{\varepsilon} /$ leblø / wi ]

* (1) à six heures du matin / ils nous emmenaient [oyi]

(2) à six heures du matin / les bleus / oui.

Ce type de phénomènes nous montrent à quel point l'écoute est une pratique liée à la subjectivité du transcripteur. On préférera cependant l'honnêteté qui, en restituant les différentes écoutes, laisse l'interprétation ouverte. L'étape de l'analyse peut en effet permettre dans certains cas de trancher. Dans l'exemple suivant ${ }^{26}$ : 
(1) sa paye / c'était sa mère qui l'en profitait

(2) sa paye / c'était sa mère qu'il en profitait

l'écoute ne permet pas d'interpréter. C'est la connaissance de l'idiolecte de la locutrice qui rend l'interprétation (2) possible : l'auteur s'est en effet aperçu qu'elle faisait un usage neutralisé du pronom personnel de la troisième personne du singulier (il qui reprend sa mère) ainsi que de nombreuses relatives en que. Le recours à l'API permet ici de ne pas

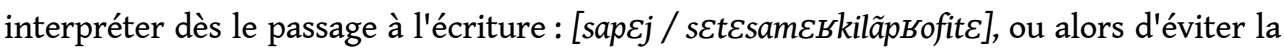
lourdeur visuelle de la double interprétation orthographique. palette de choix d'écriture, il faut savoir qu'ils ont chacun leurs avantages et leurs inconvénients ${ }^{32}$. On n'a pas jusqu'à aujourd'hui trouvé de système d'écriture parfait qui servirait pour tout type d'oral. La raison est évidente : c'est tout simplement impossible. D'une façon générale, on sait que les outils de l'écrit sont inadaptés pour écrire de l'oral et que ce qu'on gagne en précision, on le perd en lisibilité, et inversement. C'est incontournable. Regardons ce qui se passe d'un peu plus près. 
29 L'orthographe aménagée est un système qui se borne à montrer ce qui n'a pas été prononcé en utilisant différents moyens du code écrit (parenthèses et apostrophes, en général). Le principe même est gênant puisqu'il fait apparaître les formes orales comme elliptiques et incomplètes par rapport aux formes écrites. Pour se débarrasser de cette référence à l'écrit, le mieux serait de se débarrasser complètement de la graphie traditionnelle, même aménagée.

30 Basé sur un savoir implicite des règles de lecture - telle que la variation de sonorité automatique par exemple (ce qui a pour conséquence de cacher les assimilations et les dilations) - ce système permet une lecture relativement facile ${ }^{33}$. Mais, si l'orthographe aménagée offre un bon compromis entre lisibilité et précision, elle produit parfois des séquences totalement illisibles : (par)c(e) que pour [skœ] ; (de) t(ou)t(es) façons pour [ttfasõ] ; $j(e) s u(i s)$ ou $j(e) s u(i) s$ pour $\left[\int y\right]$; etc.

31 Un autre inconvénient de ce système d'écriture est qu'il peut parfois aboutir à de mauvaises interprétations de séquences: dans main(te)nant, l'orthographe suppose la chute $\mathrm{du}$ phonème $[\mathrm{t}]$, là où il $\mathrm{y}$ a nasalisation de ce même phonème. Un autre exemple de surinterprétation nous est fourni par le ne de négation après on: dans la mesure où on sait que la particule ne chute très souvent à l'oral, on peut supposer qu'elle a également chuté dans on n'y va pas. D'un autre côté, pourquoi écrirait-on systématiquement on y va pas, le locuteur ayant pu prononcer le ne de négation pour des raisons quelconques, s'ils sont fréquents dans son idiolecte par exemple. De plus, le langage de tout locuteur n'échappe pas à la variation inhérente.

32 Mal utilisée, l'orthographe aménagée peut produire des "monstres orthographiques" : alor'; deux ans e'd'mi ; y vont pas; eh s'en va ; etc. On trouve très souvent ces "trucages" dans les bandes dessinées, mais pas uniquement. Au delà du fait qu'ici l'orthographe sert à recréer des morphèmes déjà existants dans le code écrit dans les cas de $y$ et $e^{34}$, elle prétend pouvoir restituer le niveau de langue - en utilisant l'apostrophe à tort et à travers pour marquer un français dit "populaire" - ce qu'elle est bien souvent incapable de faire.

On peut aussi utiliser la graphie traditionnelle sans lui faire subir d'aménagements pour décrire des phénomènes discursifs ou même syntaxiques. Le GARS utilise ce système d'écriture parce qu'il ne s'intéresse pas à la réalisation phonique des morphèmes mais aux morphèmes eux-mêmes ${ }^{35}$. L'orthographe non aménagée peut également servir à rendre les transcriptions accessibles aux chercheurs en sciences sociales n'ayant pas de formation en linguistique.

Pour résumer, l'inconvénient majeur du système d'écriture par l'orthographe (aménagée ou non) est qu'il ne rend pas compte du registre de langue utilisé par le locuteur : il y a une perte totale de la spécificité du matériau oral. L'orthographe cache - entre autres - les phénomènes de prononciations automatiques et nous rappelle que nous ne sommes pas sortis du code écrit. D'une façon générale, si on cherche à être précis sur la prononciation, la graphie traditionnelle est systématiquement dépassée et seul l'API fournit une solution satisfaisante. Certains "trucages" orthographiques ne fonctionnent que pour les phénomènes à grande régularité, quasi-lexicalisés, tels que nan pour non ou ouais pour oui. On ne voit en effet pas quel autre moyen permettrait de restituer des

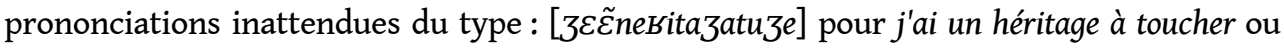
encore [mwazatãmãponjõ] pour moi j'attends mon pognon, mais également des énoncés du type : [ [pa] (ou même [ $\mathrm{p} J]$ ) pour je ne sais pas; [ivaatãnpubãtbe] pour il va attendre pour 
rentrer; etc. Ces énoncés sont loin d'être des cas isolés : ils sont le français parlé... et l'API a le mérite de donner accès à ce qui a réellement été prononcé.

L'alphabet phonétique a deux avantages fondamentaux : en marquant une différence par rapport à l'écrit, il se débarrasse des unités conventionnelles de cet écrit - non transposables à l'oral (il évite par exemple la segmentation en "mots" et respecte ainsi les groupes de souffle) et accorde un accès aux faits d'oralité plus direct et plus proche de la réalité (surtout en ce qui concerne les niveaux de langue). Il permet également de neutraliser l'interprétation - sous-jacente à la notation orthographique ${ }^{36}$ dès l'étape de l'écriture dans les cas d'homonymie ou d'ambiguïté, c'est-à-dire lorsqu'il y a « indécidabilité morphologique ${ }^{37}$. Dans l'exemple suivant:

(1) la patrie ça n'est pas toujours l'endroit où l'on est

(2) la patrie ça n'est pas toujours l'endroit où l'on naît ${ }^{38}$

l'API permet de laisser l'interprétation ouverte: [lapatßisanepatuzuslãdธwaulõne].

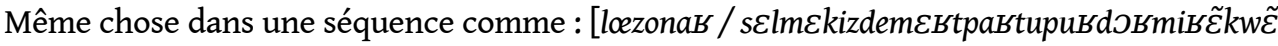
] où - dans une écriture orthographique - seule la double interprétation permet de signaler l'ambiguïté... au détriment de la lisibilité et de l'espace :

(1) le zonard / c'est l(e) mec qui s(e) démerde partout pour dormir un coin

(2) le zonard / c'est $1(\mathrm{e})$ mec i il ${ }^{39} \mathrm{~s}(\mathrm{e})$ démerde partout pour dormir un coin

Dans cet exemple, une transcription phonétique qui ferait apparaître une gémination ([ $m \varepsilon k k i])$ donne un argument, lors de l'étape de l'analyse, en faveur de l'interprétation en relative, au lieu de trancher directement lors d'une étape qui se veut descriptive.

On peut aussi reprendre l'exemple de P. Guiraud ${ }^{40}$ vous me demandez ce que ça me ferait plaisir à partir duquel il a construit son que populaire, là où il est tout aussi possible d'interpréter est-ce que réduit en [skœ] : l'écriture phonétique aurait permis de ne pas interpréter trop vite.

On peut en revanche reprocher à l'API de présenter comme des vérités scientifiques et absolues des observations qui ne peuvent être qu'approximatives en dehors du recours instrumental. Par exemple, en ce qui concerne les écoutes divergentes pour une même séquence, Leroy considère que le caractère biunivoque entre le son et le symbole "interdit bien sûr de noter une dualité ${ }^{41}$. Dans ces cas précis, "seule la graphie traditionnelle permet de le faire sans ridicule » et l'API perd alors toute sa crédibilité ${ }^{42}$. Le second reproche qu'on peut faire à ce système de notation est de voiler certains phénomènes syntaxiques, c'est-à-dire d'empêcher le repérage des morphèmes. On trouve par exemple que souvent "dissimulé" dans le discours sous la forme d'un [g] :

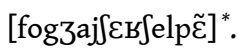

* Faut que j'aille chercher le pain.

Quant aux reproches concernant les difficultés à lire et à écrire l'alphabet phonétique, ils ne sont aujourd'hui plus soutenables.

\section{Le para-verbal (les faits prosodiques)}

Noter l'oral - même en utilisant l'API - ne suffirait pas à rendre compte totalement de l'oralité. Une transcription sans les faits prosodiques, c'est comme une chanson sans musique : dépourvue de son accompagnement, nue. L'intonation fait vivre l'oralité. Sans elle, il n'y a plus rien ; uniquement des sons, vides, inanimés.

$41 \mathrm{Au}$ delà de ces aspects "mélodiques", l'intonation a un rôle dans la structuration de l'énoncé : elle véhicule une information essentielle dans la communication. On lui accorde 
des fonctions syntaxiques ${ }^{43}$, pragmatiques et sémantiques. Beaucoup de séquences sont ambiguës sans intonation et certaines peuvent même devenir incompréhensibles. Prenons un exemple très simple et courant: ton frère vient qui peut avoir le sens d'une interrogation, d'un ordre, d'une exclamation ou tout simplement d'une affirmation ${ }^{44}$. Dans un autre exemple : on te dit tu fais ça / tu fais ça, seul le recours à l'intonation permet de restituer le sens pragmatique de l'énoncé :
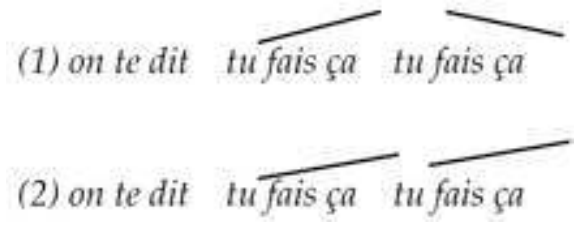

On peut grossièrement paraphraser qu'en (1) "on te demande de faire quelque chose et tu fais le contraire" alors qu'en (2) "on te demande de faire ça et puis ça, etc. (énumération)" 45 .

Même si rares sont ceux qui s'appliquent à transcrire l'intonation ${ }^{46}$, beaucoup cependant s'accordent à reconnaître son importance : « [...] il est évidemment très gênant de fournir des transcriptions de français parlé qui ne fassent pas état d'une caractéristique de l'oralité aussi importante que l'intonation. Mais l'essentiel est de ne rien mettre dans les transcriptions qui pourrait contrecarrer une éventuelle analyse intonative qu'on ajouterait ultérieurement $\aleph^{47}$. C'est qu'écoute et écriture sont extrêmement difficiles d'accès.

Reste en effet entier le problème de la transcription de l'intonation dans le cas où on ne fait pas appel aux spécialistes. Il s'agit de phénomènes très complexes et, qui plus est, peu décrits. Comme nous l'avons vu précédemment, la perception de la prosodie sans recours instrumental s'avère être encore plus embarrassante qu'elle ne l'est pour la chaîne phonématique : elle est rendue très aléatoire par notre pauvre capacité à écouter les faits prosodiques en vue de leur écriture. Il faut donc savoir rester très modeste pour la transcription de l'intonation et se contenter d'une notation approximative: signaler ce qui se passe à la jonction de groupes rythmiques (par exemple en notant par des courbes la mélodie de grands blocs) sans tenir compte des inflexions qui surviennent à l'intérieur. On peut en revanche affiner la transcription en ayant recours à des signes "orthophonétiques" (graphèmes ou phonèmes en majuscules ou en caractères gras, apostrophes, allongements vocaliques...) à l'intérieur des blocs intonatifs afin de rendre compte de phénomènes fréquents du type insistance, accent de mot, etc.

Ecrire l'intonation suppose donc un travail très long et très difficile, la "lire" ne le suppose pas moins. Selon Morel en effet $^{48}$, toute transcription des faits prosodiques aboutit forcément à des contresens de la part du lecteur. Seul le recours à l'enregistrement permet de les éviter: "Il nous faut toutefois souligner l'absolue nécessité dans laquelle nous nous sommes trouvés de travailler en permanence avec la cassette de l'enregistrement. Comme le souligne en effet Christine Leroy ${ }^{49}$, une transcription de l'oral, si minutieux que soit le codage des phénomènes prosodiques et intonatifs, ne permet que très imparfaitement l'interprétation adéquate des propos, et nous avons tous eu l'occasion de constater que seule une écoute attentive permet d'écarter des contresens faits à la simple lecture de la transcription. » 


\section{Le non-verbal (les faits extra-linguistiques)} éléments "non verbaux", ce qu'on nomme habituellement l'extralinguistique. On peut y ranger beaucoup de phénomènes : bruits divers (clics par exemple), toux, reniflements, sifflements, rires, etc.; mais également des phénomènes non sonores, tels que des regards, des grimaces, des tics, des gestes divers ${ }^{50}$. Il n'est pas question de tout retranscrire à ce niveau, d'autant plus que cela nécessiterait un enregistrement vidéo. Néanmoins, certaines indications permettent de restituer le sens pragmatique de l'énoncé. Par exemple dans [skispaslẽndãbõ / sc: (bruit)], on a l'impression que la position du complément est occupée par le bruit en l'absence duquel la séquence paraîtra incomplète. On a presqu'envie de parler de "complément non verbal". Dans de nombreux cas également, ce genre de phénomènes semblent venir ponctuer les différentes étapes de la séquence. Cela est si fréquent qu'on peut être amené à douter que des bruits comme des reniflements ou de légères toux soient davantage motivés par un besoin physiologique que par un processus de ponctuation.

Que l'écriture de l'oral ne soit pas l'oral est un fait incontournable, quels que soient les progrès technologiques et le perfectionnement des systèmes de notation dans le futur. Pour Houdebine, « il convient de se rappeler (et de souligner) que la notation phonétique la plus raffinée est elle-même un code second et qu'elle ne peut donner qu'une image de la réalité linguistique orale $»^{51}$. On peut toutefois sérieusement espérer récupérer une partie de ce "corps" ${ }^{42} q u$ 'on perd inévitablement lors du passage à l'écriture en proposant d'intégrer un maximum de paramètres ${ }^{53}$, au risque - éternel enjeu - de perdre en lisibilité.

\section{Conclusion}

« Il n'y a pas de système de transcription idéal : c'est à la fois le public visé et l'objectif de la recherche qui peuvent dicter un choix, entre les deux pôles de la fidélité et de la lisibilité $\aleph^{54}$. Pour toute étude à visée phonétique, phonologique, dialectale, et en général pour toutes les études s'intéressant à des phénomènes de prononciation, seule la transcription phonétique est opérationnelle ${ }^{55}$. Toute utilisation de la graphie traditionnelle est en ce cas impropre à décrire les faits d'oralité.

Dans d'autres perspectives de recherche, et particulièrement en syntaxe, les quelques problèmes d'ambiguïté (infinitif en "-er"/participe passé/imparfait; relatives; etc.) ne justifient pas qu'on sacrifie à la lourdeur des symboles phonétiques: la graphie traditionnelle, en ayant recours à divers moyens (multi-interprétations, notes en bas de page, mais également passages en API) peut suffire.

On peut grosso modo résumer les problèmes de choix d'écriture de l'oral en disant que plus on travaille sur de grandes unités, moins le recours à l'API est intéressant. Adapter une transcription à un but recherché ne représente pas un problème insurmontable. Le vrai problème se pose lorsqu'on parle d'élaborer des collectifs de corpus.

Peut-on ne serait-ce qu'envisager ces "collectifs de corpus oraux" ? On revient ici sur cette idée évoquée au début de l'article: établir des systèmes d'écriture communs à toutes les langues et propres à chaque champ d'étude (phonétique, lexique, syntaxe, etc.) afin de permettre une exploitation très large des données et, par la même occasion, un meilleur accès aux études comparatives. Cette idée était déjà émise il y a dix ans par 
Leroy. Pour elle en effet, "une circulation des documents est devenue réellement indispensable si l'on veut progresser rapidement dans notre connaissance de la langue orale $\aleph^{56}$. Elle évoque un peu plus loin les précautions à prendre de la part du transcripteur et du lecteur. Ce dernier doit «avoir constamment présent à l'esprit la réalité orale correspondant au texte ", ce qui revient à dire qu'il doit avoir à disposition les bandes sonores. Or, l'idée même de ces collectifs de corpus sous entend qu'on puisse y accéder facilement afin de permettre un travail en toute confiance, c'est-à-dire sans s'encombrer du document sonore authentique.

Cette idée de corpus collectifs suppose une informatisation des données tout à fait possible avec les moyens dont on dispose aujourd'hui. Le plus compliqué serait sans doute - au delà d'accepter l'idée de "partager" son corpus... - de s'accorder sur les conventions de transcription ${ }^{57}$.

Il existe aujourd'hui, en France et à l'étranger, d'importants corpus oraux mis au point dans divers domaines de la recherche linguistique ${ }^{58}$. A partir de tout ce qui existe déjà, on pourrait envisager des conventions de transcription communes à l'ensemble de la recherche et spécifiques pour chacun des champs d'étude, au lieu de continuer à produire autant de systèmes d'écriture qu'il existe de groupes de recherche, voire de chercheurs, ou - pire encore - de corpus ${ }^{59}$.

Concrètement, ce projet suppose que, dans un premier temps, chaque pays (ou communauté) "élise" des représentants dans chaque domaine qui, dans un second temps, se réuniraient pour confronter divers systèmes d'écriture afin de trouver des accords communs. Le chemin paraît d'autant plus long qu'on est conscient des problèmes que ce projet soulève : situation très "éclatée" à l'intérieur même d'un pays (pas d'accord en vue, tout au moins en France), caractère obligatoirement subjectif de toute transcription (ne serait-ce qu'au niveau de la perception), non présence des transcripteurs sur le terrain (perte d'informations pouvant cependant se suppléer par la constitution de "fiches signalétiques" très détaillées ${ }^{60}$ ), etc. Les conséquences n'en sont pas moindres: tout linguiste se verrait contraint d'abandonner son propre système d'écriture (qu'il trouvera certainement plus adéquat parce que fait sur mesure) pour se plier à des conventions très rigides ("je travaille sur telles unités, donc je dois me reconnaître dans une case prédéfinie - par exemple la morphologie - donc j'utilise telles conventions de transcription"61 ). On peut pour finir prédire un éventuel ralentissement de la production des corpus oraux, chaque chercheur préférant utiliser celui d'un autre.

Sans voir aussi loin - et avec autant d'imagination - un système d'uniformisation des conventions de transcription de l'oral pour toutes les langues et spécifiques à chaque domaine de recherche, on peut commencer à réfléchir dans ce sens. Le projet reste en effet envisageable, alors qu'il est difficilement soutenable et même inutile pour un système d'écriture qui servirait à toute fin : rien ne sert de vouloir standardiser l'écriture des données orales dans une optique "universelle" puisque cela supposerait une homogénéisation impossible de ce long et douloureux parcours - de l'enquête à l'édition face à des objectifs de recherche très hétérogènes. 


\section{BIBLIOGRAPHIE}

BARTHES, R. (1981) Le grain de la voix, Paris, Le Seuil.

BERGOUNIOUX, G. (1992) Les enquêtes de terrain en France, Langue française, 93, pp. 3-22.

BERGOUNIOUX, G., BLANCHE-BENVENISTE, C., CHEVALIER, J. C., DUMONT, C., ENCREVE, P., PERDUE, C. et SIMONI-AUREMBOU, M.-R. (1992) Table ronde : Actualité de l'enquête et des études sur l'oral, Langue française, 93, pp. 94-119.

BILGER, M. (1983) Contributions à l'analyse en grille, Recherches sur le français parlé, 4, pp. 195-215.

BLANCHE-BENVENISTE, Cl. (1993) Une description linguistique du français parlé, Le gré des langues, 5, pp. 8-29.

BLANCHE-BENVENISTE, C., BOREL, B., DEULOFEU, J., DURAND, J., GIACOMI, A., LOUFRANI, C., MEZIANE, B. et PAZERY, N. (1979) Des grilles pour le français parlé, Recherches sur le français parlé, 2, pp. 163-208.

BLANCHE-BENVENISTE, Cl. et JEANJEAN, C. (1987) Le français parlé, CNRS-INALF, Didier-Erudition. BONNARD, H. (1971) Article "Le français parlé", Le grand Larousse de la Langue Française, pp. 3983-3987.

BRANCA-ROSOFF, S. (1990) De la culture de la vigne à la cueillette des fleurs des champs, Langue française, 85, pp. 48-67.

CULIOLI, A. (1983) Pourquoi le français parlé est-il si peu étudié, Recherches sur le français parlé, 5, pp. 291-300.

DELATTRE P. (1966) Les dix intonations de base du français, French Review, 40, pp. 1-14.

DELOMIER, D. et MOREL, M.-A. (1986) Caractéristiques intonatives des incises, DRLAV, 34-35, pp. 141-160.

ESQUENET-BERNAUDIN, M. (1985) Lisibilité de l'oral : une gageure ?, Langue française, 65, pp. 17-27.

FRANÇOIS, D. (1974) Français parlé. Analyse des unités phoniques et significatives d'un corpus recueilli dans la région parisienne, Paris, SELAF.

GADET, F. (1989) Le français ordinaire, Paris, Colin.

GADET, F. et MAZIERE, F. (1986) Effets de langue orale, Langages, 81, pp. 57-73.

G.A.R.S. (1979) Recherches sur le français parlé, 2.

G.A.R.S. (1993) Guide du transcripteur - Edition de corpus de français parlé, Université de Provence, U.F.R. L.A.C.S., Département de linguistique française.

G.E.D.O., à paraître, Guide du transcripteur.

GIOVANNONI, D.-C. et SAVELLI, M.-J. (1990) Transcrire et orthographier le français parlé. De l'impossible copie à la falsification des données orales, Recherches sur le français parlé, 10, pp. 19-37.

GUIRAUD, P. (1965) Le français populaire, Que sais-je ? n 1172, Paris, PUF, 5e édition, 1986. 
HENNE, H. et REHBOCK, H. (1979) Einführung in die Gesprächsanalyse, Berlin/New York, De Gruyter. HOUDEBINE, A.-M. (1979) Pour qui, pourquoi et comment transcrire ?, Le français dans le Monde, 145 , pp 28-36.

LABOV, W. (1972a) Sociolinguistics Patterns, Philadelphia, University of Pennsylvania Press (trad. fr., 1976, Sociolinguistique, Paris, Minuit).

LABOV, W. (1972b) Language in the Inner City, Philadelphia, University of Pennsylvania Press (trad. fr., 1978, Le parler ordinaire, Paris, Minuit).

LABRIE, V. (1982) Précis de transcription de documents d'archives orales, Québec, Institut québecois de recherche sur la culture, Collection « Instruments de travail », 4.

LEON, P. (1969) Les problèmes pratiques de l'analyse intonative, Studia Phonetica, 2, Paris, Didier, pp. 25-33.

LEROY, C. (1985) La notation de l'oral, Langue française, 65, pp. 6-16.

MALDIDIER, D. (dir.), (1986) A Michel Pêcheux, Langages, 81, pp. 5-7.

MARTINIE, B. et WACHS, S. (1991) "Clochards" : un corpus de français parlé, mémoire de DEA, Vol. 1, Département des Sciences du langage, Université de Paris X-Nanterre.

MERTENS, P. (1990) Chapitre "Intonation", in C. BLANCHE-BENVENISTE, Le français parlé, Paris, CNRS, pp. 159-176.

MOCHET, M.-A. (dir.), (1983) Conventions de transcription - Document de travail, D.T.V.2, mai 1983, Projet Français des années 80 .

MOREL, M.-A. (1985) Présentation, Langue française, 65, pp. 3-5.

MOREL, M. A. (1992) Intonation et thématisation, L'Information Grammaticale, 54, pp. 26-35.

MOREL, M.-A. et RIALLAND, A. (1992) Emboîtements, autonomies, ruptures dans l'intonation française, Travaux linguistiques du Cerlico, pp. 221-243.

MOUCHON, J. (1979) La collecte du document sonore en vue de son utilisation linguistique, Le Français dans le Monde, 145, pp. 20-22.

OCHS, E. (1979) Transcription as Theory, in OCHS E. and SCHIEFFELIN B. (eds), Developmental pragmatics, New-York Academic Press, pp. 43-72.

RAINGEARD, M. et LORSCHEIDER, U. (1977) Edition d'un corpus de français parlé, Recherches sur le français parlé, 1, pp. 14-29.

SAUVAGEOT, A. (1972) Analyse du français parlé, Paris, Hachette.

STUBBS, M. (1983) Discourse Analysis. The sociolinguistic Analysis of Natural Language, Oxford, Blackwell.

THIBAULT, P. et VINCENT, D. (1988) La transcription ou la standardisation des productions orales, Linx, 18, pp. 19-32.

VIEREGGE, W. H. (1984) Problems in Phonetic transcription, Nijmegen, Inst. Fonetik.

WELKE, D. (1986) La semi-interprétativité dans les transcriptions en "analyse conversationnelle" et pragmatique linguistique : travaux américains et allemands, DRLAV,34-35, pp. 195-213. 


\section{NOTES}

1. Maldidier D. $(1986,6)$.

2. Gadet F. et Mazière F. $(1986,59)$.

3. On aura largement l'occasion d'en donner des exemples tout au long de cet article.

4. Bergounioux G. $(1992,19)$.

5. Cela pose le problème de la fiabilité des données puisque c'est la collecte des productions orales qui conditionne l'analyse. En ce sens, on peut dire que les choix méthodologiques influencent les résultats.

6. Le recours à l'image est parfois nécessaire.

7. $(1976,290)$.

8. L'idée de fiche générale nous vient du GARS (Groupe Aixois de Recherche en Syntaxe, 1993) qui parle de «fiche signalétique» mais également de Leroy C. (1985) qui proposait déjà une «carte d'identité» pour chaque corpus.

9. On reviendra en conclusion sur cette idée de "collectif de corpus", c'est-à-dire des corpus qui seraient utilisables par l'ensemble de la communauté de chercheurs dans un domaine $\mathrm{x}$ : un corpus peut-il servir à quelqu'un d'autre qu'à celui qui l'a transcrit?

10. On pense ici à certains bruits ou gestes "qui peuvent en dire long", tels que des clics, des sifflements, etc.

11. $(1974,50)$. On aimerait insister sur le fait qu'on ne cherche pas à tout décrire : on n'ira par exemple pas jusqu'à dépeindre l'aspect physique des locuteurs, en allant de la physionomie jusqu'au maquillage et en passant par les vêtements et la coiffure... tel que le proposent en analyse conversationnelle les Allemands Henne H. et Rehbock H. (1979, 62) avec leur «transcription à spectre large» aménagée dans l'optique d'établir de vastes corpus à utilisations multiples et d'aboutir tôt ou tard à une standardisation (référence trouvée dans Welke D., 1986, 206).

12. (1972a).

13. Situation à l'inverse très intéressante si l'on décide de travailler sur le degré d'hypercorrection en situation de forte surveillance par exemple.

14. Cf. l'enquête sur le "Black English Vernacular" des jeunes Noirs de Harlem (1972b). Cette enquête visait à étudier les causes de l'échec scolaire chez ces jeunes Noirs Américains, et tout particulièrement leurs difficultés dans l'apprentissage de la lecture.

15. Plus le nombre d'informateurs est élevé, plus nombreuses seront les coupures et les superpositions de paroles au sein du corpus.

16. Blanche-Benveniste C. et Jeanjean C. $(1987,96)$.

17. Seuls les résultats d'analyse sont exploités.

18. De la norme objective (telle qu'on peut l'observer) mais aussi de la norme subjective ou "évaluative" (système de valeurs).

19. Blanche-Benveniste C. et Jeanjean C. $(1987,105)$.

20. Cf. Gadet F. (1989) qui parle d'« attentes idéologiques » : « On est prêt à créditer les locuteurs favorisés de productions valorisées, à les dénier aux autres et à ne pas entendre les "fautes" chez les locuteurs légitimes » (p. 42).

21. Discours d'une même personne dans un contexte unique. Il s'agit de la variation inhérente (dite aussi intra-individuelle) : le langage de tout locuteur est sujet à la variation alors même qu'il s'adresse au(x) même(s) interlocuteur(s), dans une même situation, au même moment.

22. (1983, p. 238).

23. Ces exemples - et tous ceux qui suivront sans référence - sont attestés et issus du corpus Clochards (Martinie B. et Wachs S., 1991). 
24. Leroy C. (1985) utilise ces exemples pour dénoncer le caractère illusoirement scientifique de l'API (on y reviendra un peu plus loin).

25. [3lœfE] peut ici s'interpréter comme un mot valise entre "je logeais" et "je créchais".

26. Gadet F. (1989, 42).

27. Tels que les analyseurs de mélodie, le mingogramme, les logiciels spécialisés, etc.

28. Leroy C. $(1985,8)$.

29. On a vu ce qui s'est passé avec François D. (1974) qui livrait il y a vingt ans une première transcription phonétique "brute", c'est-à-dire ne reposant sur aucune hypothèse d'analyse précise : «cette transcription a ensuite été revue en fonction des problèmes qui se posaient et, éventuellement, affinée après nouvelles auditions des enregistrements» (p. 50), ce qui sous entend une nouvelle écoute pour des points précis relatifs à la recherche. Donc, encore une fois, une écriture inévitablement orientée.

30. Welke D. $(1986,202)$.

31. C'est-à-dire tout ce qui relève du contexte situationnel et qui peut jouer sur l'oralité.

32. On peut ici mentionner un autre système de transcription reconnu: les configurations en grilles du GARS. Une des spécificités majeures de l'oral par rapport à l'écrit étant de ne pas se dérouler linéairement (hésitations, ruptures, reformulations, etc.), la notation en grilles vise à casser cette linéarité écrite difficile à lire en présentant visuellement - et orthographiquement le discours oral. Cf. Blanche-Benveniste C. et alii (1979) ainsi que Bilger M. (1983).

33. Au delà du "camouflage" des phénomènes phonétiques, l'orthographe aménagée - en reposant sur l'hypothèse d'un savoir linguistique partagé - interdit toute possibilité de corpus collectifs (cf. note 9) : tout le monde ne partage pas le même savoir.

34. Des morphèmes auxquels l'oeil pourrait se raccrocher.

35. Le GARS fait forcément ses transcriptions en vue d'une exploitation grammaticale des corpus. Dans les seuls cas de productions "marginales" (régionalismes, enfants en bas âge, etc.), il peut avoir besoin de recourir à l'écriture phonétique ou encore d'aménager la graphie. Cf. à ce sujet Leroy C. (1985) qui considère que l'utilisation de la graphie traditionnelle pour rendre compte de productions d'enfants en bas âge ne peut qu'aboutir à de l'« adultomorphisme ».

36. Déjà en 1972, Sauvageot A. présentait l'API tout simplement comme le «seul procédé applicable pour ne pas être induit en erreur par la graphie» (p. 8).

37. Expression de Gadet F. et Mazière F. $(1986,60)$.

38. Cet exemple est emprunté à Blanche-Benveniste C. et Jeanjean C. $(1987,105)$.

39. On sait (de par cette connaissance commune des règles implicites de lecture) que le $l$ final de la troisième personne du pronom personnel sujet ne se prononce pas devant consonne : écrire $i(l)$ ou $i(l s)$ serait tout aussi ridicule que d'écrire fusi(l) ou $c u(l)$, par exemple.

40. $(1986,7)$.

41. $(1985,12)$ où elle se sert des exemples alors et là-bas.

42. Faire figurer une multi-interprétation en alphabet phonétique n'est gênant, à nos yeux, en aucun point, dans la mesure où il doit être admis une fois pour toute que l'écoute ne peut pas, par définition, être débarrassée de toute subjectivité.

43. Cf. Morel M. A. (1992) qui propose un modèle syntaxique basé sur l'observation de phénomènes intonatifs.

44. Exemple qu'utilise Bonnard H. (1971) pour montrer qu'à l'oral le recours constant à l'intonation sert à «suppléer les marques segmentales » (p. 3985).

45. Avec une intonation moins descendante - voire même en plateau - sur le troisième segment de l'énoncé, on peut interpréter (1) encore différemment : "on te demande de faire quelque chose et tu ne discutes pas".

46. Comme cela nécessite de l'entraînement et de l'appareillage, on réserve volontiers ce travail à la compétence de spécialistes... (Delattre P., Delomier D., Léon P., Mertens P., Morel M. A., Rialland A., etc.) 
47. Blanche-Benveniste C. et Jeanjean C. $(1987,130)$.

48. $(1985,3)$.

49. $(1985,7-8)$.

50. Il paraît préférable de noter ces phénomènes en parallèle avec le verbal pour préserver le synchronisme des événements.

51. $(1979,30)$.

52. On fait ici référence à Barthes R. (1981) : « (...) ce qui se perd dans la transcription, c'est tout simplement le corps (...)» (p. 11).

53. Directement pertinents pour le discours oral.

54. Gadet F. (1989, 44).

55. «Quand il s'agit de représenter du patois qui n'a pas à se conformer à un usage reçu, d'un humble patois sans littérature, l'orthographe académique dont on l'affuble jure autant qu'un habit noir sur le dos d'un paysan " (La Revue des patois gallo-romans, 1887, 2è année, nº, compte rendu de Charles Joret, Flore populaire de la Normandie, Paris, Maisonneuve, par Dr. A. Bos), référence trouvée dans Branca-Rosoff S. $(1990,64)$.

56. $(1985,8)$.

57. Même si tous les chercheurs s'intéressant aux discours oraux sont d'accord pour affirmer incontournables les différentes étapes de la transcription, ils sont bien loin de s'accorder sur les systèmes de notation. Pourquoi adopter un système plutôt qu'un autre ? Ce désaccord est remarquablement illustré par les nombreux débats que cela soulève. D'un autre côté, la présence même de ces débats montre à quel point les problèmes d'édition de l'oral sont partagés par un grand nombre de chercheurs, ce qui pourrait peut-être constituer le premier pas vers une mise en commun des données orales.

58. Plus spécifiquement en analyse du discours et en syntaxe de l'oral en France. Cf. le GARS ainsi que le GEDO (Groupe d'Etude sur les Données Orales) qui est entrain de mettre au point un «Guide du transcripteur » (à paraître).

59. L'homogénéité du système de transcription chez un même linguiste n'est pas si évidente.

60. Cf. note 8 .

61. Cette catégorisation en "cases" suppose qu'à chaque champ d'étude corresponde la même réalité pour tous : problème de frontières dont la délimitation commune peut suffire à ce type de projet.

\section{RÉSUMÉS}

Je m'intéresse à l'incontournable paradoxe de l'accessibilité des discours oraux en vue de leur analyse : étudier l'oral suppose qu'on le rende visible et l'écrire présuppose qu'on quitte le domaine de l'oralité...

En décrivant avec minutie toutes les étapes qui jalonnent le long et pénible parcours qui va de l'enquête à l'édition des données orales (recueil, écoute, notation) j'ai voulu montrer que ces différentes étapes sont essentielles - mais malheureusement peu reconnues pour telles - et déterminantes pour l'analyse des discours oraux : ce parcours ne se fait pas au hasard mais se construit en fonction des objectifs de recherche. Le travail d'analyse ne commence donc pas à l'écriture mais s'élabore lentement dès le recueil des données: la transcription n'est pas réductible à un élément empirique mais devient un élément constitutif de l'analyse proprement 
dite.

A partir de ce constat, j'ai voulu aborder l'idée de constitution de corpus collectifs, utilisables par tout chercheur intéressé. Ce projet est envisageable dans des domaines de recherche précis (phonétique, syntaxe, lexique, etc.). Concrètement cela suppose un accord international sur des conventions de transcription - propres à chacun des champs d'étude et communs à toutes les langues. Avoir à disposition ce type de corpus permettrait un élargissement extrêmement intéressant de l'exploitation des données orales par l'ensemble de la recherche et, à un autre niveau, un meilleur accès aux études comparatives. Je reste cependant consciente que la mise en place d'un tel projet ne peut se faire sans problèmes et conséquences.

I am interested in the inevitable paradox of the accessibility of oral discourse with a view to its analysis : studying the spoken word supposes that we make it visible and writing it down means that we leave the world of speech...

In describing meticulously the stages that mark the difficult and long progression from collating to writing down the oral data, I wanted to show that these different stages are essential - but unfortunately not recognised as such - and can determine oral discourse analysis: this progression does not occur in a haphazard way but is constructed according to the research objectives. Consequently, the work of analysis does not begin with writing down but elaborates itself very slowly as soon as data is collected: transcription is not simplified to an empirical element but becomes a constituent element of the analysis itself.

From these observations, I wanted to confront the idea of the constitution of collective corpora that can be used by any researcher. This project is entirely feasible for closely defined research (phonetics, syntax, lexicon, etc.). In real terms, this presupposes an international agreement on the methods of transcription appropriate to each objective and common to all languages. To have this kind of corpus would allow a very interesting widening of the use of oral data for the general research and - on another level - better access to comparative studies. However I am aware that such a project can not be set up without problems and consequences.

\section{AUTEUR}

\section{SANDRINE WACHS}

